\title{
Quantum Metrology with Strongly Interacting Spin Systems
}

\author{
Hengyun Zhou $\odot,{ }^{1, \dagger}$ Joonhee Choi,${ }^{1,2, \dagger}$ Soonwon Choi $\odot,{ }^{3}$ Renate Landig, ${ }^{1}$ Alexander M. Douglas, ${ }^{1}$ Junichi Isoya, ${ }^{4}$ \\ Fedor Jelezko, ${ }^{5}$ Shinobu Onoda $\odot,{ }^{6}$ Hitoshi Sumiya $\odot,{ }^{7}$ Paola Cappellaro, ${ }^{8}$ Helena S. Knowles, ${ }^{1,9}$ \\ Hongkun Park ${ }^{1,10}$ and Mikhail D. Lukin ${ }^{1, *}$, \\ ${ }^{1}$ Department of Physics, Harvard University, Cambridge, Massachusetts 02138, USA \\ ${ }^{2}$ School of Engineering and Applied Sciences, Harvard University, Cambridge, Massachusetts 02138, USA \\ ${ }^{3}$ Department of Physics, University of California Berkeley, Berkeley, California 94720, USA \\ ${ }^{4}$ Tsukuba Research Center for Energy Materials Science, Faculty of Pure and Applied Sciences, \\ University of Tsukuba, Tsukuba, Ibaraki 305-8573, Japan \\ ${ }^{5}$ Institut für Quantenoptik, Universität Ulm, 89081 Ulm, Germany \\ ${ }^{6}$ Takasaki Advanced Radiation Research Institute, 1233 Watanuki, Takasaki, Gunma 370-1292, Japan \\ ${ }^{7}$ Sumitomo Electric Industries Ltd., Itami, Hyougo, 664-0016, Japan \\ ${ }^{8}$ Research Laboratory of Electronics and Department of Nuclear Science and Engineering, \\ Massachusetts Institute of Technology, Cambridge, Massachusetts 02139, USA \\ ${ }^{9}$ Cavendish Laboratory, University of Cambridge, JJ Thomson Avenue, \\ Cambridge CB3 OHE, United Kingdom \\ ${ }^{10}$ Department of Chemistry and Chemical Biology, Harvard University, \\ Cambridge, Massachusetts 02138, USA
}

(Received 23 January 2020; revised 23 March 2020; accepted 6 May 2020; published 2 July 2020)

\begin{abstract}
Quantum metrology is a powerful tool for explorations of fundamental physical phenomena and applications in material science and biochemical analysis. While in principle the sensitivity can be improved by increasing the density of sensing particles, in practice this improvement is severely hindered by interactions between them. Here, using a dense ensemble of interacting electronic spins in diamond, we demonstrate a novel approach to quantum metrology to surpass such limitations. It is based on a new method of robust quantum control, which allows us to simultaneously suppress the undesired effects associated with spin-spin interactions, disorder, and control imperfections, enabling a fivefold enhancement in coherence time compared to state-of-the-art control sequences. Combined with optimal spin state initialization and readout directions, this allows us to achieve an ac magnetic field sensitivity well beyond the previous limit imposed by interactions, opening a new regime of high-sensitivity solid-state ensemble magnetometers.
\end{abstract}

DOI: 10.1103/PhysRevX.10.031003

\section{INTRODUCTION}

Electronic spins associated with color centers in diamond have recently emerged as a promising platform for nanoscale precision sensing and imaging, with superior sensitivity and spatial resolution [1-7]. A common approach to improving sensitivity of such quantum systems is to utilize a dense ensemble of individual sensors and take advantage of parallel averaging. However, beyond a certain density, undesired interactions between

\footnotetext{
${ }^{*}$ Corresponding author. lukin@physics.harvard.edu

${ }^{\dagger}$ These authors contributed equally to this work.

Published by the American Physical Society under the terms of the Creative Commons Attribution 4.0 International license. Further distribution of this work must maintain attribution to the author(s) and the published article's title, journal citation, and DOI.
}

Subject Areas: Quantum Physics

sensors result in a rapid decay of the ensemble coherence $[8,9]$, limiting the overall sensitivity of the quantum system [Fig. 1(a)]. Moreover, in practice, disorder and control errors further deteriorate coherence and metrological sensitivity of such interacting many-body quantum systems. Over the past few decades, pulsed control techniques realizing dynamical decoupling and motional averaging have been developed and deployed to manipulate interacting ensembles of quantum systems. While extremely successful in the context of nuclear magnetic resonance (NMR), magnetic resonance imaging [10-15], and atomic gas magnetometers [16], the efficacy of these techniques is severely limited in the presence of strong disorder and other imperfections. Therefore, these techniques are not directly applicable to quantum sensors based on electronic spin ensembles [17], where such effects are prominent.

In this article, we demonstrate a new approach for quantum sensing with disordered interacting spin 

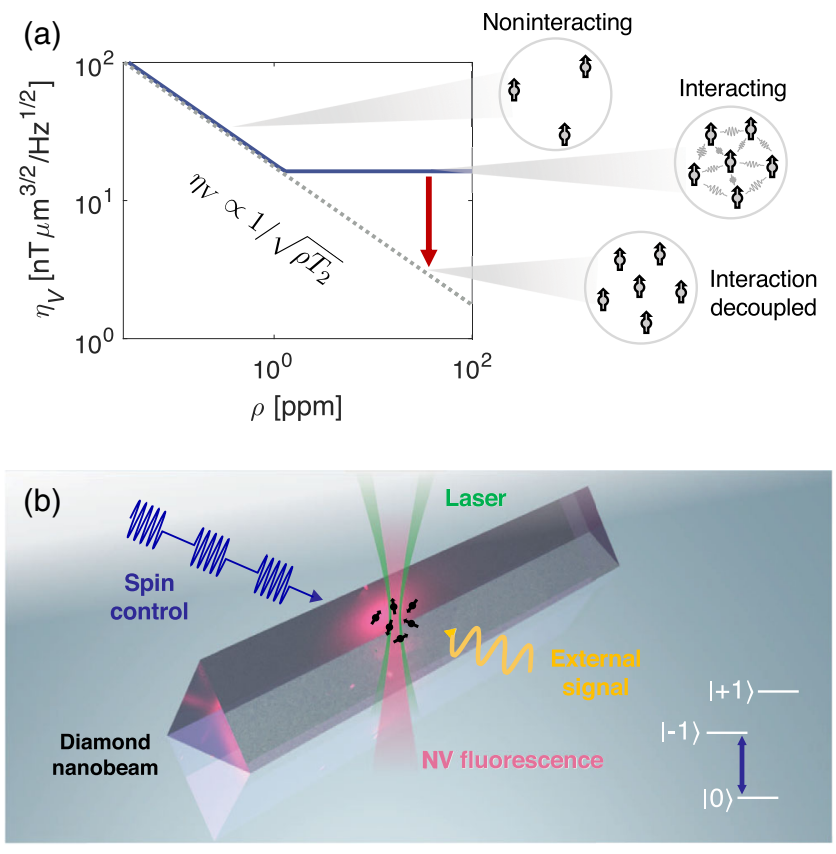

FIG. 1. Interaction limit to spin ensemble quantum sensing. (a) Volume-normalized magnetic field sensitivity as a function of total spin density. The dashed line denotes the standard quantum limit scaling and the solid curve shows the behavior when interactions between spins are taken into account for the typical readout efficiency factor $C \approx 0.0028$ [27]. The sensitivity plateaus beyond a critical density due to a coherence time reduction. Robust interaction decoupling (red arrow) allows us to break the interaction limit. (b) Illustration of the black diamond nanobeam used as a spin ensemble quantum sensor. Microwave and optical excitation are delivered to the NV spins to control and read out their spin states, and an ac magnetic field is used as a target sensing signal. The inset shows the three magnetic sublevels, $|0\rangle$ and $| \pm 1\rangle$, in the ground state of NV centers, where two levels $|0,-1\rangle$ are addressed using resonant microwave driving. All measurements are performed at room temperature under ambient conditions.

ensembles. Our method uses periodic pulsed manipulation (Floquet engineering) of a spin ensemble [10] to detect an external signal of interest with high sensitivity, while simultaneously decoupling the effects of interactions and disorder, and being fault tolerant against the leading-order imperfections arising from finite pulse durations and experimental control errors. Specifically, we introduce a set of simple rules imposed on the pulse sequence for disordered, interacting systems and a new, generalized picture of ac-field sensing that is essential in the interacting regime. These go beyond existing dynamical decoupling techniques for noninteracting spin systems $[6,18-24]$ and low-disorder NMR systems [10-15,25], allowing us to design and implement a new sequence, DROID-60 (Disorder-RObust Interaction-Decoupling), that breaks the sensitivity limit on ac sensing imposed by spin-spin interactions for the first time.

\section{EXPERIMENTAL SYSTEM}

Our experimental system consists of a dense electronic spin ensemble of nitrogen-vacancy (NV) centers in diamond [26], as shown in Fig. 1(b). NV centers exhibit long-lived spin coherence even at room temperature and are excellent sensors of magnetic fields, electric fields, pressure, and temperature [5,7,27-37]. Our sample has a high density of NV centers ( $\sim 15 \mathrm{ppm}$; see Supplemental Material [17] for details), with long-range magnetic dipolar interactions between the spins as well as strong on-site disorder originating from other paramagnetic impurities, inhomogeneous strain in the diamond lattice, and local electric fields [26]. The bulk diamond is etched into a nanobeam to improve control homogeneity and confine the probing volume to $V=8.1(9) \times 10^{-3} \mu \mathrm{m}^{3}$ [17]. The NV center ground state is an electronic $S=1$ spin, and we apply a static magnetic field to isolate an ensemble of effective two-level systems formed of NV centers with the same crystallographic orientation. We initialize and detect the spin states optically and use resonant microwave excitation to drive coherent spin dynamics. See Supplemental Material [17] for further details of the measurement sequences.

\section{ROBUST INTERACTION DECOUPLING}

Spin echo measurements [38] reveal that the coherence decay time $T_{2}$ in our dense NV ensemble is limited to only $1.0 \mu \mathrm{s}$ [Fig. 2(c), gray crosses]. The conventional method to extend $T_{2}$ beyond the spin echo is the XY- 8 dynamical decoupling sequence [18], consisting of equally spaced $\pi$ pulses along the $\hat{x}$ and $\hat{y}$ axes [Fig. 2(a), top row]. In our system, however, the XY-8 sequence only provides a small improvement $\left(T_{2}=1.6 \mu \mathrm{s}\right)$ [Fig. 2(c), blue circles], since the XY-8 $T_{2}$ is limited by strong spin-spin interactions, which are not affected by $\pi$ rotations (see Supplemental Material [17] for density scaling measurements which further confirm that the XY- $8 T_{2}$ is interaction limited).

In order to significantly extend $T_{2}$ in the presence of interactions and control imperfections, we use a novel approach to design pulse sequences [39]. We model our spin system, including the control fields and the external ac magnetic field target signal, by the Hamiltonian $[17,26]$

$$
H=H_{s}+H_{\Omega}(t)+H_{\mathrm{ac}}(t),
$$

where the internal system Hamiltonian is $H_{s}=\sum_{i} h_{i} S_{i}^{z}+$ $\sum_{i j}\left(J_{i j} / r_{i j}^{3}\right)\left(S_{i}^{x} S_{j}^{x}+S_{i}^{y} S_{j}^{y}-S_{i}^{z} S_{j}^{z}\right), \quad$ global spin-control pulses are given by $H_{\Omega}(t)=\sum_{i}\left[\Omega_{i}^{x}(t) S_{i}^{x}+\Omega_{i}^{y}(t) S_{i}^{y}\right]$, and the external target signal is $H_{\mathrm{ac}}(t)=$ $\gamma_{\mathrm{NV}} B_{\mathrm{ac}} \cos \left(2 \pi f_{\mathrm{ac}} t-\phi\right) \sum_{i} S_{i}^{z}$. Here, $S_{i}^{\mu}(\mu=x, y, z)$ are spin-1/2 operators, $h_{i}$ is a random on-site disorder potential with zero mean that follows a Gaussian distribution with standard deviation $W=(2 \pi) 4.0 \mathrm{MHz}, J_{i j} / r_{i j}^{3}$ is the anisotropic dipolar interaction strength between two spins of the 


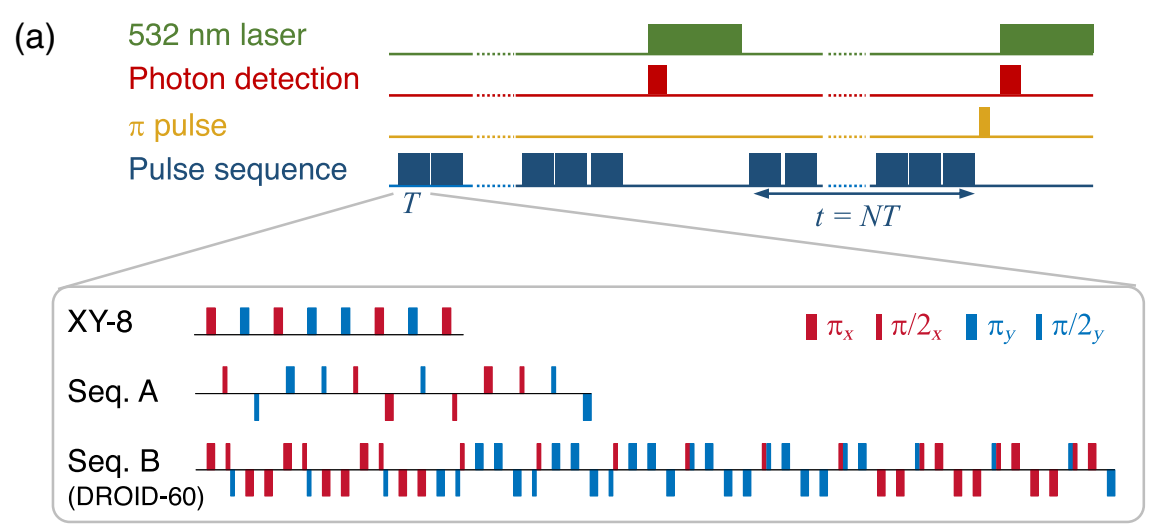

(b)

Mutually correcting robust rotations
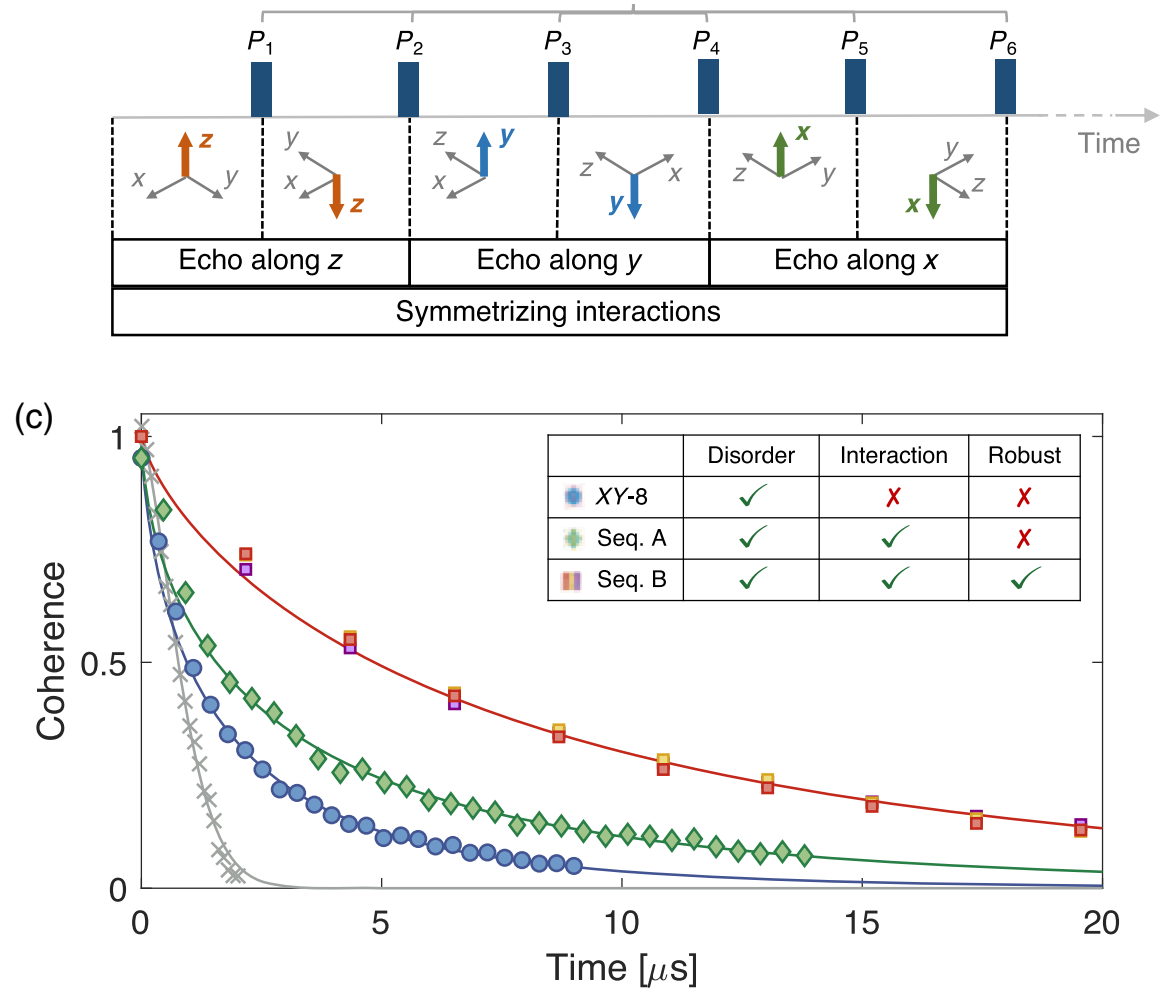

FIG. 2. Robust dynamical decoupling. (a) Measurement protocol. NV centers are initialized using pulsed laser excitation at $532 \mathrm{~nm}$ (green trace) and read out through emitted photons detected by a single photon counting module (red trace). We perform $N$ repetitions of a sensing sequence unit of length $T$ (blue trace) and repeat the same measurement with an additional $\pi$ pulse (yellow trace) acting on the NV centers for differential readout of the spin state [17]. The box illustrates the details of different pulse sequences, XY-8, Seq. A, and Seq. B (DROID-60), composed of $\pi / 2$ and $\pi$ rotations along $\hat{x}$ and $\hat{y}$ axes. Bars above (below) the line indicate driving along positive (negative) axis directions. (b) Key concepts for sequence design. The sequence is described by pulses $P_{i}$ and the time-dependent frame transformation of the system between the pulses. We highlight the orientation of each rotated frame by the axis that points along the $\hat{z}$ axis of the fixed external reference frame. Decoupling sequences are designed by imposing average Hamiltonian conditions on the evolution of the highlighted axis. For example, the effects of disorder can be canceled by implementing an echolike evolution, $+\hat{\mu} \rightarrow-\hat{\mu}$, where $\mu=x, y, z$ (top row), and interactions are symmetrized by equal evolution in each of the $\hat{x}, \hat{y}$, and $\hat{z}$ axes in the transformed frames (bottom row). Additionally, the pulse sequence is designed to mutually correct rotation angle errors and finite pulse duration effects [17]. (c) Experimental performance of different sequences with their respective decoupling features (inset). We fit the decoherence profile with a stretched exponential $e^{-\left(t / T_{2}\right)^{\alpha}}$ (solid curves) to extract the coherence time $T_{2}$ for each sequence [17]. A simple spin echo (gray crosses), XY-8 (blue circles), and Seq. A (green diamonds) show $T_{2}=0.98(2), 1.6(1)$, and 2.8(1) $\mu$ s with $\alpha=1.5(1), 0.66(2)$, and 0.61(3), respectively. DROID-60 (squares), designed to correct for all leading-order effects of interactions, disorder, and control imperfections, gives $T_{2}=7.9(2) \mu$ s with $\alpha=0.75(2)$. We confirm that its coherence time is independent of the initial state prepared along $\hat{x}, \hat{y}$, and $\hat{z}$ axes, as shown in red, yellow, and purple, respectively. All sequences have pulse spacing $\tau=25 \mathrm{~ns}$ and $\pi$-pulse width $\tau_{\pi}=20 \mathrm{~ns}$. 
same crystallographic orientation at a distance $r_{i j}$, with strength $J=(2 \pi) 35 \mathrm{kHz}$ at a typical separation of $11 \mathrm{~nm}$, $\Omega_{i}^{x, y}(t)$ are the global control amplitudes exhibiting weak position dependence due to spatial field inhomogeneities, $\gamma_{\mathrm{NV}}$ is the gyromagnetic ratio of the NV center, and $B_{\mathrm{ac}}, f_{\mathrm{ac}}$, and $\phi$ are the amplitude, frequency, and phase of the target ac signal, respectively.

Our approach employs average Hamiltonian theory to engineer the system evolution through pulsed periodic manipulation of the spins $[10,38,40]$. A sequence composed of $n$ equidistant control pulses $P_{k},(k=1,2, \ldots, n)$ with spacing $\tau$ defines a unitary time-evolution operator $\mathcal{U}(T)=P_{k} e^{-i H_{s} \tau} \cdots P_{1} e^{-i H_{s} \tau}$ over the Floquet period $T$. If the pulse spacing $\tau$ is much shorter than the timescales of the system Hamiltonian $(\tau \ll(1 / W), 1 / J)$, the unitary operator $\mathcal{U}(T)$ can effectively be approximated by a timeindependent average Hamiltonian as $\mathcal{U}(T) \approx e^{-i H_{\mathrm{av}} T}$, with $H_{\mathrm{av}}=(1 / T) \sum_{k=1}^{n} \tilde{H}_{k}$, and $\tilde{H}_{k}=\left(P_{k-1} \cdots P_{1}\right)^{\dagger} \times$ $H_{s}\left(P_{k-1} \cdots P_{1}\right), H_{1}=H_{s}$. Motivated by this picture, we develop a pulse sequence which generates a desirable form of $H_{\mathrm{av}}$ from the $H_{s}$ intrinsic to the system.

Hamiltonian engineering can be understood as the result of a sequence of frame transformations (also known as toggling frame transformations) which rotate the spin operators in the interaction picture [Fig. 2(b)]: for example, a $\pi$ pulse flips $S_{i}^{z} \rightarrow-S_{i}^{z}$, while a $\pi / 2$ pulse rotates $S_{i}^{z} \rightarrow$ $\pm S_{i}^{x, y}$ depending on the rotation axis. Importantly, the average Hamiltonian is uniquely specified by such toggling frame transformations of the $S^{z}$ operator [17], resulting in simple decoupling conditions that facilitate the procedure to find desired pulse sequences.

For example, any pulse sequence in which the transformed $S^{z}$ operator spends equal time along the positive and negative direction for each axis-effectively producing a spin echo along all three axes-suppresses the on-site disorder Hamiltonian. Similarly, in order to symmetrize the dipolar interaction into a Heisenberg interaction Hamiltonian (where polarized states are eigenstates and coherence is preserved [41]), we require that the transformed $S^{z}$ operator spend an equal amount of time in each direction $\hat{x}, \hat{y}, \hat{z}[11,17]$. Furthermore, we can prioritize one condition over another to find a pulse sequence that better suits a given system; since disorder is dominant in our spin ensemble $(W \gg J)$, we perform the echo operation more frequently than interaction symmetrization.

In realistic situations, the above strategy will be affected by various imperfections, such as disorder and interactions acting during the finite pulse durations and errors of each control pulse, resulting in imperfections $\delta H_{\text {av }}$ to the target effective Hamiltonian, $H_{\text {eff }}=H_{\mathrm{av}}+\delta H_{\mathrm{av}}$. Indeed, if we simply design a pulse sequence that decouples disorder and interactions only in the ideal pulse limit [Seq. A in Fig. 2(a)], we see only a marginal increase in coherence time compared to XY-8, yielding $T_{2}=2.8 \mu \mathrm{s}$ [Fig. 2(c), green diamonds]. A careful examination of Seq. A reveals that pulse-related imperfections play a dominant role in the dynamics, illustrating the importance of robust sequence design [17].

To address this key challenge, a number of strategies have been proposed that aim to cancel or control the undesired Hamiltonian terms acting during the finite pulse duration [12-15,23,24,42,43]; however, a simple and systematic approach to treating the imperfections in a general setting, particularly for interacting systems, is still lacking. Remarkably, we find that the transformations of the $S^{z}$ operator during the free-evolution intervals are in fact sufficient to predict and suppress the errors during pulse rotations. This is a consequence of the fact that the unwanted residual Hamiltonian acting within the finite pulse duration can be uniquely determined by the two toggling-frame Hamiltonians on either side of the control pulse [17]. This insight motivates us to describe the $S^{z}$ operator transformations using the matrix $\mathbf{F}=\left[F_{\mu, k}\right]=$ $2 \operatorname{Tr}\left[S^{\mu} \tilde{S}_{k}^{z}\right], \mu=\hat{x}, \hat{y}, \hat{z}$, where $\tilde{S}_{k}^{z}$ is the transformed spin operator within the $k$ th free-evolution period. Crucially, this allows us to construct a simple set of algebraic conditions imposed on the matrix $\mathbf{F}$ to formalize the above decoupling rules, enabling not only the suppression of disorder and interaction effects during free-evolution periods, but also the cancellation of dominant imperfections arising from finite pulses as well as rotation angle errors (see Supplemental Material [17] for the exact expressions of decoupling conditions). This also enables system-customized design for optimal ac-field sensing, taking into account the energetic hierarchy between on-site disorder, spin-spin interactions, and control imperfections in a given system, significantly extending beyond existing techniques.

By satisfying these rules, we can thus systematically generate robust pulse sequences that to first order yield a pure Heisenberg Hamiltonian with $\delta H_{\mathrm{av}}=0$. The DROID60 sequence (Disorder-RObust Interaction-Decoupling with 60 pulses per period), as shown in Fig. 2(a), is an example of a robust pulse sequence designed with our formalism [17]. Because of its robustness against all leading-order effects, it shows a significant extension of coherence time compared to the sequences described above, reaching $T_{2}=7.9 \mu \mathrm{s}$ [Fig. 2(c), squares]. Moreover, the coherence time is independent of the initial state.

\section{OPTIMAL SENSING WITH INTERACTION DECOUPLING}

We now apply this method to quantum sensing, where our goal is to robustly engineer the dynamics of the spin ensemble to be sensitive to the target sensing signal. ac magnetic field sensing typically uses periodic inversions of the spin operator between $S^{z}$ and $-S^{z}$ in the interaction picture, driven by a train of equidistant $\pi$ pulses at a separation of $\left(1 / 2 f_{\mathrm{ac}}\right)$. This modulation causes cumulative precession of the sensor spin when the ac-field sign change coincides with the frame inversion, resulting in high sensitivity to a signal field at $f_{\text {ac }}[6,44,45]$. Our interaction-decoupling sequences explore all three frame directions $S^{x}, S^{y}, S^{z}$, and ac selectivity requires synchronized 
periodic frame inversions in each of the three axes, while preserving the desired $H_{\text {av }}$ and suppressing $\delta H_{\text {av }}$ to maintain long coherence times.

In Fig. 3(a) we illustrate how this is achieved in DROID60. The pulses lead to periodic changes in the sign and

(a)
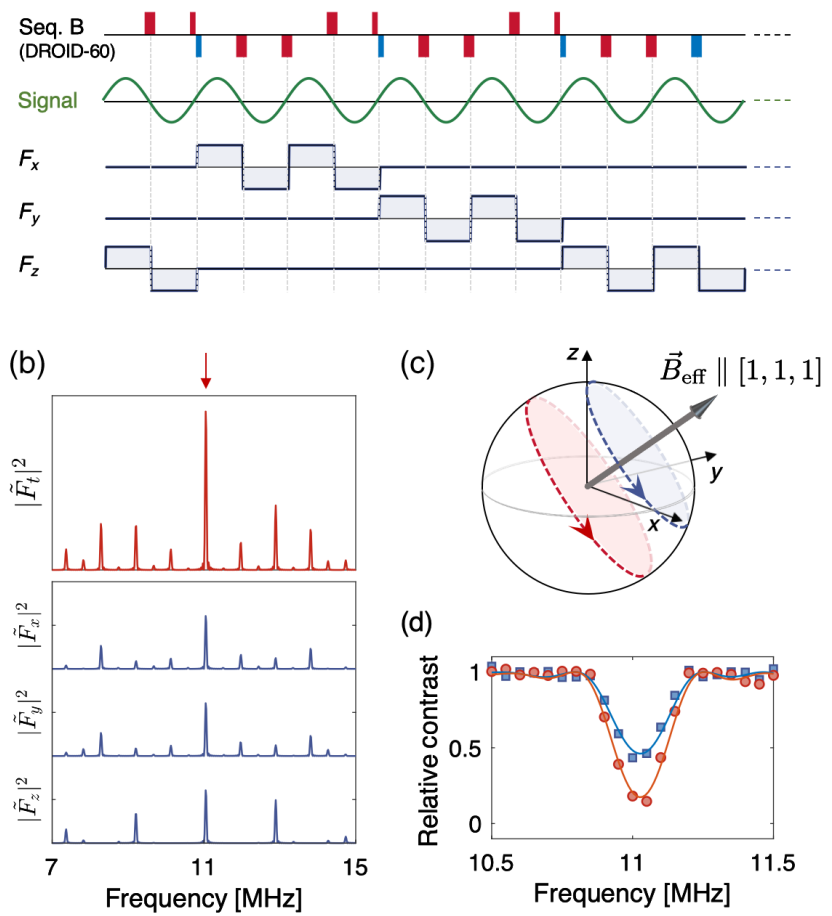

FIG. 3. Optimal sensing with unconventional spin state preparation. (a) Pulse sequence (top row) and three-axis time-domain modulation functions (blue solid curves) for the first 14 freeevolution times of DROID-60. Red and blue bars in DROID-60 indicate rotation pulses as defined in Fig. 2(a). The modulation period along each axis is synchronized to an ac sensing signal (green curve). (b) Frequency-domain modulation function $\left|\tilde{F}_{x, y, z}(f)\right|^{2}$ for DROID-60, with pulse spacing $\tau=25 \mathrm{~ns}$ and $\pi$-pulse width $\tau_{\pi}=20 \mathrm{~ns}$. The total strength $\left|\tilde{F}_{t}(f)\right|^{2}$ is obtained from individual axis amplitudes $\tilde{F}_{x, y, z}(f)$ considering their relative phases in the frequency domain [17]. The principal resonance is highlighted by a red arrow, yielding maximum sensitivity. (c) Illustration of the effective magnetic field created by DROID-60 at the principal resonance. In the average Hamiltonian picture, the $\hat{z}$-direction sensing field in the external reference frame transforms to the [1,1,1]-direction field $\vec{B}_{\text {eff }}$ in the effective spin frame with a reduced strength [17]. Optimal sensitivity is achieved by initializing the spins into the plane perpendicular to the effective magnetic field direction. This optimal state preparation allows the spins to precess along the trajectory with the largest contrast (red dashed line). For comparison, the precession evolution for initialization to the conventional $\hat{x}$ axis is shown as a blue dashed line. (d) Sensing resonance spectra near the principal resonance. The optimal initialization (red) shows greater contrast than the $\hat{x}$-axis initialization (blue). Markers indicate experimental data and solid lines denote theoretical predictions calculated from the frequencydomain modulation functions [17]. orientation of the interaction-picture $S^{z}$ operator, depicted by the time-domain modulation functions for each axis direction, $F_{x}, F_{y}$, and $F_{z}$. The detailed resonance properties of the pulse sequence can be characterized by the Fourier transforms $\tilde{F}_{\mu}(f)=\left|\tilde{F}_{\mu}(f)\right| e^{-i \tilde{\phi}_{\mu}(f)}$ of $F_{\mu}$ for $\mu=x, y, z$, where $\tilde{\phi}_{\mu}(f)$ is the spectral phase for a given axis $\mu$. Figure 3(b) shows the calculated spectral intensities along different axes, $\left|\tilde{F}_{x}(f)\right|^{2},\left|\tilde{F}_{y}(f)\right|^{2}$, and $\left|\tilde{F}_{z}(f)\right|^{2}$, as well as the total intensity $\left|\tilde{F}_{t}(f)\right|^{2}[17]$. At the dominant resonance of the total intensity [red arrow in Fig. 3(b)], all three axes exhibit a phase-locked periodic sign modulation [Fig. 3(a)], leading to constructive phase accumulation and high sensitivity. With this phase-locked sign modulation, the response of DROID-60 to the phase alignment between a target ac signal field and the sequence is similar to the standard XY-8 sequence, and thus it can be used to sense both phase-coherent and random phase signals $[6,17]$.

In order to intuitively understand our sensing protocol and quantify its sensitivity, we generalize the average Hamiltonian analysis to incorporate ac signal fields, finding [17]

$$
\begin{aligned}
H_{\mathrm{av}, \mathrm{ac}} & =\gamma_{\mathrm{NV}} B_{\mathrm{ac}} \sum_{i} \operatorname{Re}\left[\sum_{\mu=x, y, z} \tilde{F}_{\mu}\left(f_{\mathrm{ac}}\right) S_{i}^{\mu} e^{i \phi}\right] \\
& =\gamma_{\mathrm{NV}} \vec{B}_{\mathrm{eff}} \cdot \sum_{i} \vec{S}_{i}
\end{aligned}
$$

where $\vec{B}_{\text {eff }}$ is an effective magnetic field vector in the interaction picture which appears static to the driven spins. This allows a simple interpretation of our scheme: the spins undergo a precession around $\vec{B}_{\text {eff }}$, with the field orientation and magnitude determined by the frequency-domain modulation functions $\tilde{F}_{\mu}$ and $\left|\tilde{F}_{t}\right|$, respectively. For conventional sequences with $\vec{B}_{\text {eff }} \| \hat{z}$, the requirements for interaction symmetrization dictate that only $1 / 3$ of the total sensing time can be spent along any given axis, resulting in a significant loss of sensitivity. In contrast, for our optimal DROID-60, the signal at the principal resonance $f_{\text {ac }}$ gives rise to $\left|\tilde{F}_{x}\right|=\left|\tilde{F}_{y}\right|=\left|\tilde{F}_{z}\right|$, with $\tilde{\phi}_{x}=\tilde{\phi}_{y}=\tilde{\phi}_{z}$, leading to $\vec{B}_{\text {eff }} \propto\left[\frac{1}{3}, \frac{1}{3}, \frac{1}{3}\right]$, with the total strength $\left|\vec{B}_{\text {eff }}\right|$ reduced by a factor of only $1 / \sqrt{3}$. This reduction is fundamental and, in fact, close to optimal [39], given the requirements to suppress the effects of spin-spin interactions via symmetrization. However, despite the reduction, the sensitivity is still improved because the coherence time is extended by interaction suppression. Moreover, the sensitivity to the signal $B_{\text {ac }}$ is maximized when the spins are initialized perpendicular to the $\vec{B}_{\text {eff }}$ direction-to allow the largest precession orbit [Fig. 3(c)]—and the corresponding optimal readout requires an unconventional rotation axis $[-1,1,0]$ and angle $\arccos (\sqrt{2 / 3})$ to bring the precession plane parallel to the $\hat{z}$ axis. Indeed, as shown in Fig. 3(d), we observe a larger contrast when spins are initialized in an optimal direction along $[1,1,-2]$, compared to an initialization in the conventional $\hat{x}$ direction. 


\section{DEMONSTRATION OF SENSITIVITY ENHANCEMENT}

We now proceed to characterize the ac magnetic field sensitivity, defined as the minimum detectable signal amplitude per unit time, $\eta=\left(\sigma_{S} /\left|d S / d B_{\text {ac }}\right|\right)$. Here, $S$ is the spin contrast, $\sigma_{S}$ is the uncertainty of $S$ for one second of averaging, and $\left|d S / d B_{\text {ac }}\right|$ is the gradient of $S$ with respect to the field amplitude $B_{\text {ac }}$.

Figure 4(a) shows the measured contrast $S$ as a function of $B_{\mathrm{ac}}$ under optimal conditions for DROID-60 and the conventional sequence XY-8, where we choose acquisition parameters for each of the two sequences that optimize their respective absolute sensitivities [17]. We find that the spin contrast shows faster oscillations and a significantly steeper maximum slope under DROID-60, indicating that it is more sensitive to the external signal than XY-8. This is due to a combination of optimal state preparation and readout schemes as well as significantly improved coherence times, despite a reduced effective signal strength $\left|\vec{B}_{\text {eff }}\right|$. In Fig. 4(b), we show the sensitivity scaling with phase accumulation time, for a fixed signal frequency and integration time, finding good agreement with the theoretical prediction [17]. We find that the volume-normalized sensitivity $\eta_{V}=\eta \sqrt{V}$ of DROID-60, designed with our optimal sensing approach, reaches more than $40 \%$ improvement in sensitivity over the conventional XY-8 sequence. Moreover, we find that the improvement persists even for sizable (10\%) systematic rotation angle (a)

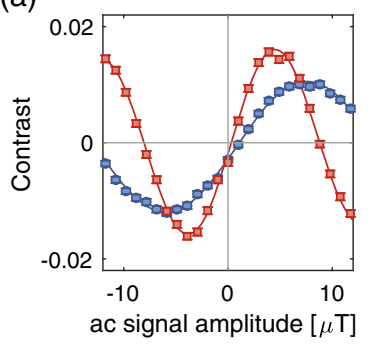

(b)

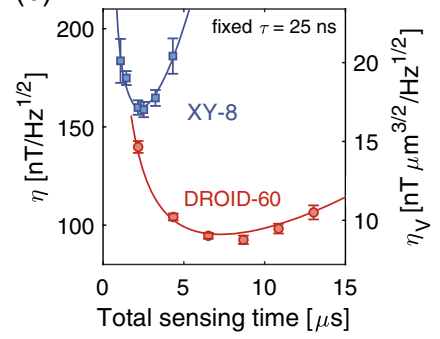

FIG. 4. Demonstration of sensitivity enhancement. (a) Observed spin contrast as a function of ac magnetic field strength, for the XY-8 sequence (blue) and for DROID-60 (red). The fit is a sinusoidal oscillation with an exponentially decaying profile [17]. Sequence B shows a steeper slope at zero field, indicating that it is more sensitive than XY-8 to the external field. The interrogation times, $t=2.16 \mu \mathrm{s}$ for XY-8 and $t=6.52 \mu \mathrm{s}$ for DROID-60, are independently optimized to achieve maximal sensitivity [17]. (b) Extracted absolute sensitivity $\eta$ and volume-normalized sensitivity $\eta_{V}=\eta \sqrt{V}$ with sensing volume $V=8.1(9) \times$ $10^{-3} \mu \mathrm{m}^{3}$ as a function of phase accumulation time $t$ (error bars are given for $\eta$ ). The pulse spacing $\tau$ is fixed at $25 \mathrm{~ns}$ to detect the ac signal oscillating at frequency $11 \mathrm{MHz}$ [see Fig. 3(d)]. A comparison of the two sequences at their respective optimal sensing times reveals that DROID-60 outperforms XY-8 by $\sim 40 \%$. The solid lines indicate the theoretical sensitivity scaling using the independently estimated sensor characteristics [17]. errors, confirming the sequence robustness [17]. With these enhancements, we demonstrate $\eta_{V}=8.3(9)$ $\mathrm{nT} \mu \mathrm{m}^{3 / 2} / \sqrt{\mathrm{Hz}}$ for DROID-60, among the best volumenormalized ac sensitivities for solid-state magnetometers measured thus far [46] (see Supplemental Material [17] for a direct comparison).

\section{DISCUSSION}

Our work establishes a novel approach to quantum sensing by utilizing robust interaction decoupling and provides the first demonstration of a solid-state ensemble quantum sensor surpassing the interaction limit. Additionally, our design formalism and generalized effective field picture are also directly applicable to robust dcfield sensing. For example, for dc-field sensing with interacting spins, the well-known Waugh-Huber-Haberlen (WAHUHA) pulse sequence [11] not only cancels interactions but also transforms an external dc field along the $z$ axis to an effective sensing field in the $[1,1,1]$ direction, and thus the initialization and readout directions should be chosen in the same way as in DROID-60. While our approach already yields a significant improvement in sensitivity, the $T_{2}$ reached here is still shorter than the depolarization time $T_{1} \sim 100 \mu \mathrm{s}$ in our dense spin ensemble. This $T_{2}$ is likely limited by waveform distortions in control pulses and higher-order terms in the average Hamiltonian analysis. The former can be mitigated by waveform engineering [47,48], and the latter by sequence symmetrization [12] or disorder reduction via spin-bath engineering [49-51]. Moreover, the reported sensitivity value here is characterized with a relatively low readout efficiency of $C \sim 0.003$, limited by both charge state instabilities of the interacting NV ensemble [52] and the low quantum efficiency of the photodetector used in this study [17], indicating room for substantial future improvements. Together with new diamond growth techniques [53-57], double-quantum magnetometry [51,58,59], and improved photon collection $[46,60]$, these improvements may push the volume-normalized sensitivity to single-digit picotesla level in a $\mu \mathrm{m}^{3}$ volume [17], opening the door to many applications, such as high-sensitivity nanoscale NMR [3,4,61-64] and investigations of strongly correlated condensed matter systems [2]. Beyond applications to diamond magnetometry, the robust sequence design presented in this work can be extended to engineer a broad class of many-body Hamiltonians $[39,41]$ in a wide variety of quantum hardware platforms, providing a useful tool for quantum information processing [65], simulation [66-68], and metrology [69-71].

All data needed to evaluate the conclusions are present in the paper and/or the Supplemental Material [17]. Additional data related to this paper may be requested from the authors [72]. 


\section{ACKNOWLEDGMENTS}

The authors thank W.W. Ho, A. Omran, C. Ramananthan, T. Sumarac, and E. Urbach for helpful discussions, A. Sushko for experimental assistance, and F. Machado and L. Martin for critical reading of the manuscript. This work was supported in part by Center for Ultracold Atoms, Vannevar Bush Faculty Fellowship Program, ARO MURI, DARPA DRINQS, Moore Foundation GBMF-4306, Samsung Fellowship, Miller Institute for Basic Research in Science, NSF PHY1506284, Japan Society for the Promotion of Science KAKENHI (No. 26246001), EU (FP7, Horizons 2020, ERC), DFG, SNSF, and BMBF. H.Z., J.C., S.C., and M.D.L. conceived the idea. H.Z., J.C., and S. C. performed the theoretical analysis and pulse sequence design. H. Z., J. C., R. L., A. M. D., and H. S. K. performed the experiment and data analysis. J. I., F. J., S. O., and H. S. provided the sample. H.Z., J.C., and H.S. K. wrote the manuscript, with input from all authors. M. D. L. and H. P. supervised the research.

[1] D. DeMille, J. M. Doyle, and A. O. Sushkov, Probing the Frontiers of Particle Physics with Tabletop-Scale Experiments, Science 357, 990 (2017).

[2] F. Casola, T. van der Sar, and A. Yacoby, Probing Condensed Matter Physics with Magnetometry Based on Nitrogen-Vacancy Centres in Diamond, Nat. Rev. Mater. 3, 17088 (2018).

[3] N. Aslam, M. Pfender, P. Neumann, R. Reuter, A. Zappe, F. F. De Oliveira, A. Denisenko, H. Sumiya, S. Onoda, J. Isoya, and J. Wrachtrup, Nanoscale Nuclear Magnetic Resonance with Chemical Resolution, Science 357, 67 (2017).

[4] D. R. Glenn, D. B. Bucher, J. Lee, M. D. Lukin, H. Park, and R. L. Walsworth, High-Resolution Magnetic Resonance Spectroscopy Using a Solid-State Spin Sensor, Nature (London) 555, 351 (2018).

[5] R. Schirhagl, K. Chang, M. Loretz, and C. L. Degen, Nitrogen-Vacancy Centers in Diamond: Nanoscale Sensors for Physics and Biology, Annu. Rev. Phys. Chem. 65, 83 (2014).

[6] C. L. Degen, F. Reinhard, and P. Cappellaro, Quantum Sensing, Rev. Mod. Phys. 89, 035002 (2017).

[7] L. Rondin, J.-P. Tetienne, T. Hingant, J.-F. Roch, P. Maletinsky, and V. Jacques, Magnetometry with NitrogenVacancy Defects in Diamond, Rep. Prog. Phys. 77, 056503 (2014).

[8] M. W. Mitchell, Sensor Self-Interaction, Scale-Invariant Spin Dynamics, and the $\hbar$ Limit of Field Sensing, arXiv:1904.01528.

[9] M. W. Mitchell and S. P. Alvarez, Colloquium: Quantum Limits to the Energy Resolution of Magnetic Field Sensors, Rev. Mod. Phys. 92, 021001 (2020).

[10] U. Haeberlen and J. S. Waugh, Coherent Averaging Effects in Magnetic Resonance, Phys. Rev. 175, 453 (1968).
[11] J. S. Waugh, L. M. Huber, and U. Haeberlen, Approach to High-Resolution NMR in Solids, Phys. Rev. Lett. 20, 180 (1968).

[12] P. Mansfield, Symmetrized Pulse Sequences in High Resolution NMR in Solids, J. Phys. C 4, 1444 (1971).

[13] D. P. Burum and W. K. Rhim, Analysis of Multiple Pulse NMR in Solids. III, J. Chem. Phys. 71, 944 (1979).

[14] B. F. Chmelka and A. Pines, Some Developments in Nuclear Magnetic Resonance of Solids, Science 246, 71 (1989).

[15] D. G. Cory, J. B. Miller, and A. N. Garroway, TimeSuspension Multiple-Pulse Sequences: Applications to Solid-State Imaging, J. Magn. Reson. 90, 205 (1990).

[16] J. C. Allred, R. N. Lyman, T. W. Kornack, and M. V. Romalis, High-Sensitivity Atomic Magnetometer Unaffected by Spin-Exchange Relaxation, Phys. Rev. Lett. 89, 130801 (2002).

[17] See Supplemental Material at http://link.aps.org/ supplemental/10.1103/PhysRevX.10.031003 for the details of the experimental setup and parameters, pulse sequence design, and sensitivity estimation for ac magnetometry.

[18] D. Suter and G. A. Álvarez, Colloquium: Protecting Quantum Information against Environmental Noise, Rev. Mod. Phys. 88, 041001 (2016).

[19] L. Viola, E. Knill, and S. Lloyd, Dynamical Decoupling of Open Quantum Systems, Phys. Rev. Lett. 82, 2417 (1999).

[20] G. De Lange, Z. H. Wang, D. Ristè, V. V. Dobrovitski, and R. Hanson, Universal Dynamical Decoupling of a Single Solid-State Spin from a Spin Bath, Science 330, 60 (2010).

[21] A. Ajoy, G. A. Álvarez, and D. Suter, Optimal Pulse Spacing for Dynamical Decoupling in the Presence of a Purely Dephasing Spin Bath, Phys. Rev. A 83, 032303 (2011).

[22] D. Farfurnik, A. Jarmola, L. M. Pham, Z. H. Wang, V. V. Dobrovitski, R. L. Walsworth, D. Budker, and N. Bar-Gill, Optimizing a Dynamical Decoupling Protocol for SolidState Electronic Spin Ensembles in Diamond, Phys. Rev. B 92, 060301(R) (2015).

[23] J. E. Lang, J. Casanova, Z.-Y. Wang, M. B. Plenio, and T. S. Monteiro, Enhanced Resolution in Nanoscale NMR via Quantum Sensing with Pulses of Finite Duration, Phys. Rev. Applied 7, 054009 (2017).

[24] G. T. Genov, D. Schraft, N. V. Vitanov, and T. Halfmann, Arbitrarily Accurate Pulse Sequences for Robust Dynamical Decoupling, Phys. Rev. Lett. 118, 133202 (2017).

[25] K. I. O. Ben'Attar, D. Farfurnik, and N. Bar-Gill, Hamiltonian Engineering of General Two-Body Spin-1/2 Interactions, Phys. Rev. Research 2, 013061 (2020).

[26] G. Kucsko, S. Choi, J. Choi, P. C. Maurer, H. Zhou, R. Landig, H. Sumiya, S. Onoda, J. Isoya, F. Jelezko, E. Demler, N. Y. Yao, and M. D. Lukin, Critical Thermalization of a Disordered Dipolar Spin System in Diamond, Phys. Rev. Lett. 121, 023601 (2018).

[27] J. M. Taylor, P. Cappellaro, L. Childress, L. Jiang, D. Budker, P. R. Hemmer, A. Yacoby, R. Walsworth, and M. D. Lukin, High-Sensitivity Diamond Magnetometer with Nanoscale Resolution, Nat. Phys. 4, 810 (2008).

[28] C. L. Degen, Scanning Magnetic Field Microscope with a Diamond Single-Spin Sensor, Appl. Phys. Lett. 92, 243111 (2008). 
[29] F. Dolde, H. Fedder, M. W. Doherty, T. Nöbauer, F. Rempp, G. Balasubramanian, T. Wolf, F. Reinhard, L. C. L. Hollenberg, F. Jelezko, and J. Wrachtrup, Electric-Field Sensing Using Single Diamond Spins, Nat. Phys. 7, 459 (2011).

[30] M. W. Doherty, V. V. Struzhkin, D. A. Simpson, L. P. McGuinness, Y. Meng, A. Stacey, T. J. Karle, R. J. Hemley, N. B. Manson, L. C. L. Hollenberg, and S. Prawer, Electronic Properties and Metrology Applications of the Diamond $\mathrm{NV}^{-}$Center under Pressure, Phys. Rev. Lett. 112, 047601 (2014).

[31] S. Hsieh, P. Bhattacharyya, C. Zu, T. Mittiga, T. J. Smart, F. Machado, B. Kobrin, T. O. Höhn, N. Z. Rui, M. Kamrani, S. Chatterjee, S. Choi, M. Zaletel, V. V. Struzhkin, J. E. Moore, V. I. Levitas, R. Jeanloz, and N. Y. Yao, Imaging Stress and Magnetism at High Pressures Using a Nanoscale Quantum Sensor, Science 366, 1349 (2019).

[32] M. Lesik, T. Plisson, L. Toraille, J. Renaud, F. Occelli, M. Schmidt, O. Salord, A. Delobbe, T. Debuisschert, L. Rondin, P. Loubeyre, and J.-F. Roch, Magnetic Measurements on Micrometer-Sized Samples under High Pressure Using Designed NV Centers, Science 366, 1359 (2019).

[33] K. Y. Yip, K. O. Ho, K. Y. Yu, Y. Chen, W. Zhang, S. Kasahara, Y. Mizukami, T. Shibauchi, Y. Matsuda, S. K. Goh, and S. Yang, Measuring Magnetic Field Texture in Correlated Electron Systems under Extreme Conditions, Science 366, 1355 (2019).

[34] Y.-X. Shang, F. Hong, J.-H. Dai, Hui-Yu, Y.-N. Lu, E.-K. Liu, X.-H. Yu, G.-Q. Liu, and X.-Y. Pan, Magnetic Sensing inside a Diamond Anvil Cell via Nitrogen-Vacancy Center Spins, Chin. Phys. Lett. 36, 086201 (2019).

[35] G. Kucsko, P. C. Maurer, N. Y. Yao, M. Kubo, H. J. Noh, P. K. Lo, H. Park, and M. D. Lukin, Nanometre-Scale Thermometry in a Living Cell, Nature (London) 500, 54 (2013).

[36] P. Neumann, I. Jakobi, F. Dolde, C. Burk, R. Reuter, G. Waldherr, J. Honert, T. Wolf, A. Brunner, J. H. Shim, D. Suter, H. Sumiya, J. Isoya, and J. Wrachtrup, HighPrecision Nanoscale Temperature Sensing Using Single Defects in Diamond, Nano Lett. 13, 2738 (2013).

[37] D. M. Toyli, C. F. de las Casas, D. J. Christle, V. V. Dobrovitski, and D.D. Awschalom, Fluorescence Thermometry Enhanced by the Quantum Coherence of Single Spins in Diamond., Proc. Natl. Acad. Sci. U.S.A. 110, 8417 (2013).

[38] C. P. Slichter, Principles of Magnetic Resonance (Springer Science \& Business Media, Berlin/Heidelberg, 2013), Vol. 1.

[39] J. Choi, H. Zhou, H. S. Knowles, R. Landig, S. Choi, and M. D. Lukin, preceding paper, Robust Dynamic Hamiltonian Engineering of Many-Body Spin Systems, Phys. Rev. X 10, 031002 (2020).

[40] M. H. Levitt, Spin Dynamics: Basics of Nuclear Magnetic Resonance (John Wiley \& Sons, New York, 2001), p. 686.

[41] S. Choi, N. Y. Yao, and M. D. Lukin, Dynamical Engineering of Interactions in Qudit Ensembles, Phys. Rev. Lett. 119, 183603 (2017).

[42] J. E. Lang, R. B. Liu, and T. S. Monteiro, DynamicalDecoupling-Based Quantum Sensing: Floquet Spectroscopy, Phys. Rev. X 5, 041016 (2015).
[43] Z.-Y. Wang, J. E. Lang, S. Schmitt, J. Lang, J. Casanova, L. McGuinness, T. S. Monteiro, F. Jelezko, and M. B. Plenio, Randomization of Pulse Phases for Unambiguous and Robust Quantum Sensing, Phys. Rev. Lett. 122, 200403 (2019).

[44] J. Bylander, S. Gustavsson, F. Yan, F. Yoshihara, K. Harrabi, G. Fitch, D. G. Cory, Y. Nakamura, J.-S. Tsai, and W. D. Oliver, Noise Spectroscopy through Dynamical Decoupling with a Superconducting Flux Qubit, Nat. Phys. 7, 565 (2011).

[45] V. M. Frey, S. Mavadia, L. M. Norris, W. de Ferranti, D. Lucarelli, L. Viola, and M. J. Biercuk, Application of Optimal Band-Limited Control Protocols to Quantum Noise Sensing, Nat. Commun. 8, 2189 (2017).

[46] T. Wolf, P. Neumann, K. Nakamura, H. Sumiya, T. Ohshima, J. Isoya, and J. Wrachtrup, Subpicotesla Diamond Magnetometry, Phys. Rev. X 5, 041001 (2015).

[47] N. Khaneja, T. Reiss, C. Kehlet, T. Schulte-Herbrüggen, and S. J. Glaser, Optimal Control of Coupled Spin Dynamics: Design of NMR Pulse Sequences by Gradient Ascent Algorithms, J. Magn. Reson. 172, 296 (2005).

[48] P. Doria, T. Calarco, and S. Montangero, Optimal Control Technique for Many-Body Quantum Dynamics, Phys. Rev. Lett. 106, 190501 (2011).

[49] G. de Lange, T. van der Sar, Z.-H. Wang, R. Hanson, V. Dobrovitski, and M. Blok, Controlling the Quantum Dynamics of a Mesoscopic Spin Bath in Diamond, Sci. Rep. 2, 382 (2012).

[50] H. S. Knowles, D. M. Kara, and M. Atatüre, Observing Bulk Diamond Spin Coherence in High-Purity Nanodiamonds, Nat. Mater. 13, 21 (2014).

[51] E. Bauch, C. A. Hart, J. M. Schloss, M. J. Turner, J. F. Barry, P. Kehayias, S. Singh, and R. L. Walsworth, Ultralong Dephasing Times in Solid-State Spin Ensembles via Quantum Control, Phys. Rev. X 8, 031025 (2018).

[52] J. Choi, S. Choi, G. Kucsko, P. C. Maurer, B. J. Shields, H. Sumiya, S. Onoda, J. Isoya, E. Demler, F. Jelezko, N. Y. Yao, and M. D. Lukin, Depolarization Dynamics in a Strongly Interacting Solid-State Spin Ensemble, Phys. Rev. Lett. 118, 093601 (2017).

[53] A. M. Edmonds, U.F.S. D'Haenens-Johansson, R. J. Cruddace, M. E. Newton, K.-M. C. Fu, C. Santori, R. G. Beausoleil, D. J. Twitchen, and M. L. Markham, Production of Oriented Nitrogen-Vacancy Color Centers in Synthetic Diamond, Phys. Rev. B 86, 035201 (2012).

[54] T. Fukui, Y. Doi, T. Miyazaki, Y. Miyamoto, H. Kato, T. Matsumoto, T. Makino, S. Yamasaki, R. Morimoto, N. Tokuda, M. Hatano, Y. Sakagawa, H. Morishita, T. Tashima, S. Miwa, Y. Suzuki, and N. Mizuochi, Perfect Selective Alignment of Nitrogen-Vacancy Centers in Diamond, Appl. Phys. Express 7, 055201 (2014).

[55] J. Michl, T. Teraji, S. Zaiser, I. Jakobi, G. Waldherr, F. Dolde, P. Neumann, M. W. Doherty, N. B. Manson, J. Isoya, and J. Wrachtrup, Perfect Alignment and Preferential Orientation of Nitrogen-Vacancy Centers during Chemical Vapor Deposition Diamond Growth on (111) Surfaces, Appl. Phys. Lett. 104, 102407 (2014).

[56] M. Lesik, T. Plays, A. Tallaire, J. Achard, O. Brinza, L. William, M. Chipaux, L. Toraille, T. Debuisschert, A. Gicquel, J. Roch, and V. Jacques, Preferential orientation 
of NV defects in CVD diamond films grown on (113)oriented substrates, Diam. Relat. Mater. 56, 47 (2015).

[57] T. R. Eichhorn, C. A. McLellan, and A. C. B. Jayich, Optimizing the Formation of Depth-Confined Nitrogen Vacancy Center Spin Ensembles in Diamond for Quantum Sensing, Phys. Rev. Mater. 3, 113802 (2019).

[58] K. Fang, V. M. Acosta, C. Santori, Z. Huang, K. M. Itoh, H. Watanabe, S. Shikata, and R. G. Beausoleil, High-Sensitivity Magnetometry Based on Quantum Beats in Diamond Nitrogen-Vacancy Centers, Phys. Rev. Lett. 110, 130802 (2013).

[59] H. J. Mamin, M. H. Sherwood, M. Kim, C. T. Rettner, K. Ohno, D. D. Awschalom, and D. Rugar, Multipulse DoubleQuantum Magnetometry with Near-Surface NitrogenVacancy Centers, Phys. Rev. Lett. 113, 030803 (2014).

[60] D. Hopper, H. Shulevitz, and L. Bassett, Spin Readout Techniques of the Nitrogen-Vacancy Center in Diamond, Micromachines 9, 437 (2018).

[61] D. Rugar, H. J. Mamin, M. H. Sherwood, M. Kim, C. T. Rettner, K. Ohno, and D. D. Awschalom, Proton Magnetic Resonance Imaging Using a Nitrogen-Vacancy Spin Sensor, Nat. Nanotechnol. 10, 120 (2015).

[62] T. Häberle, D. Schmid-Lorch, F. Reinhard, and J. Wrachtrup, Nanoscale Nuclear Magnetic Imaging with Chemical Contrast, Nat. Nanotechnol. 10, 125 (2015).

[63] S. J. DeVience, L. M. Pham, I. Lovchinsky, A. O. Sushkov, N. Bar-Gill, C. Belthangady, F. Casola, M. Corbett, H. Zhang, M. Lukin, H. Park, A. Yacoby, and R. L. Walsworth, Nanoscale NMR Spectroscopy and Imaging of Multiple Nuclear Species, Nat. Nanotechnol. 10, 129 (2015).
[64] F. Shi, Q. Zhang, P. Wang, H. Sun, J. Wang, X. Rong, M. Chen, C. Ju, F. Reinhard, H. Chen, J. Wrachtrup, J. Wang, and J. Du, Single-Protein Spin Resonance Spectroscopy under Ambient Conditions, Science 347, 1135 (2015).

[65] J. Stajic, The Future of Quantum Information Processing, Science 339, 1163 (2013).

[66] J. Zhang, G. Pagano, P. W. Hess, A. Kyprianidis, P. Becker, H. Kaplan, A. V. Gorshkov, Z. X. Gong, and C. Monroe, Observation of a Many-Body Dynamical Phase Transition with a 53-Qubit Quantum Simulator, Nature (London) 551, 601 (2017).

[67] S. Choi, J. Choi, R. Landig, G. Kucsko, H. Zhou, J. Isoya, F. Jelezko, S. Onoda, H. Sumiya, V. Khemani, C. von Keyserlingk, N. Y. Yao, E. Demler, and M. D. Lukin, Observation of Discrete Time-Crystalline Order in a Disordered Dipolar Many-Body System, Nature (London) 543, 221 (2017).

[68] K. X. Wei, P. Peng, O. Shtanko, I. Marvian, S. Lloyd, C. Ramanathan, and P. Cappellaro, Emergent Prethermalization Signatures in Out-of-Time Ordered Correlations, Phys. Rev. Lett. 123, 090605 (2019).

[69] M. Kitagawa and M. Ueda, Squeezed Spin States, Phys. Rev. A 47, 5138 (1993).

[70] P. Cappellaro and M. D. Lukin, Quantum Correlation in Disordered Spin Systems: Applications to Magnetic Sensing, Phys. Rev. A 80, 032311 (2009).

[71] S. Choi, N. Y. Yao, and M. D. Lukin, Quantum Metrology Based on Strongly Correlated Matter, arXiv:1801.00042.

[72] lukin@physics.harvard.edu 